\title{
Optimal Energy Consumption to Extend the Lifetime of Wireless Sensor Networks
}

\author{
${ }^{1} N$. Thangadurai and ${ }^{2}$ R. Dhanasekaran \\ ${ }^{1}$ Research Scholar, Research \& Development Centre, Bharathiar University, Coimbatore, India \\ ${ }^{2}$ Director-Research, Syed Ammal Engineering College, Ramanathapuram, India
}

Received 2013-04-16, Revised 2013-05-09; Accepted 2012-05-27

\begin{abstract}
Recent advances in Wireless Sensor Networks (WSNs) enable us to develop minimum energy consumption clustering algorithms. The WSNs can be used for various applications like battle field, disaster areas and military applications. The most important issue in WSNs is the limited battery of sensor nodes. When sensor nodes are placed in a field, it is difficult to replace their batteries. To minimize the energy consumption of nodes, WSNs introduce various protocols and algorithms. Among the techniques, the genetic based energy efficient clustering algorithms are used to minimize the energy consumption of nodes and to extend the network lifetime. Genetic Algorithm (GA) is an adaptive method for search and optimize problems and the genetic process is based on biological organisms. Clustering is an effective way for reducing energy consumption of a sensor nodes as well as the cost of transmission. The proposed genetic algorithm based clustering protocol provide better efficiency in terms of delay, energy consumption. Here we simulated the proposed protocol by using network simulator ns 2.34 and compared with other existing protocols.
\end{abstract}

Keywords: Genetic Algorithm, Clustering, LEACH, LEACH-C

\section{INTRODUCTION}

Wireless Sensor Networks become more important for many applications (Zhu et al., 2009). Figure 1 shows a sensor network having large number of sensor nodes deployed very close to each other. In WSNs, sensor nodes have limited battery, when it is placed in the specific field. It is difficult to replace their batteries or to supply additional energy (Guo et al., 2011). If a node consumes completely its energy, whole network gets disconnected. High population of nodes and minimum node energy consumption are managed by clustering mechanism. Data aggregation is used to eliminate data redundancy and minimize the communication overload.

The position of the sensor nodes does not need to be pre-determined (Akyildiz et al., 2002). Routing algorithms having self-organizing capabilities and consist of different sensors namely seismic, low sampling rate magnetic, thermal, visual, infrared and acoustic sensors. WSNs were used in various applications like military applications, environment monitoring and detecting wild life in a dense forest area. Designing of sensor nodes having some constraints like the small size, low weight, energy consumption, multi functional, communicate with short distance and low price and minimum transmission cost. Most known applications are target tracking, habit monitoring, surveillance and security (Bandyopadhyay and Coyle, 2003). Cluster based approach is useful for environment monitoring. WSN is the combination of wireless communication and environmental perception. It is a special form of wireless ADHOC network. This can construct the network without any infrastructure. Energy efficient routing algorithms are mainly divided in to the following categories. (1) Reduction of the communication energy consumption by adopting multi-hop transmission strategy. (2) Balancing the network load by adopting the cluster-based routing protocols and optimizing the location of cluster head. (3) Adopting the sleep and wake-up mechanism to avoid the unnecessary energy consumption. Corresponding Author: N. Thangadurai, Research Scholar, Research \& Development Centre, Bharathiar University, Coimbatore, India 


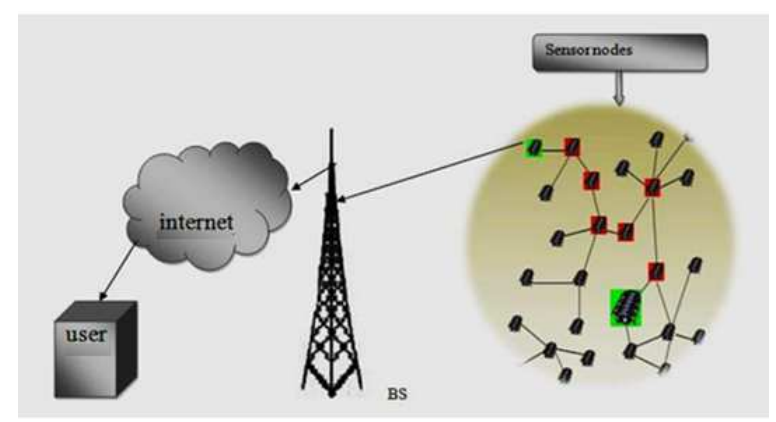

Fig. 1. WSN Architecture

The energy of a node is very limited; the maximum lifetime of WSN plays an important role to design the routing protocols. The efficient routing protocol plays an important role for packet transmission and also considers the Network balance.

\section{GENETIC ALGORITHM}

This section provides basic idea on GAs and defines its terms and describes how a simple GA works. Genetic algorithms are search algorithms based on natural selection and recombination process (Guo et al., 2011). They attempt to find a solution to the problem at hand by manipulating a population of candidates. The population is evaluated and the best solutions are selected to reproduce to form the next generation. In number of generations, good traits dominate the total population, resulting in better quality of the solutions. The basic ideas in GAs are Darwinian evolution. We follow Goldberg (1989) and restrict the notion of a building block to the shortest schemata that contributes to the global optimum.

\section{RELATED WORK}

Genetic algorithm based clustering protocols Low Energy Adaptive Clustering Hierarchy (LEACH) algorithm for clustering protocol was proposed by Heinzelman et al. (2000). In LEACH the network area is divided in to several clusters and each cluster has a Cluster Head $(\mathrm{CH})$ and cluster members transmit data to their own sink (Guo et al., 2011). Extension of LEACH is known as LEACH-C protocol. Younis and Fahmy (2004) proposed a protocol namely HEED. In TEEN and APTEEN protocols, the network area is divided in to several clusters and among the clusters, one node will be introduced as a head of the cluster and the task of these cluster heads are to collect the data sent from the nodes of the cluster members, mixing the data and forward those data to the sink. LEACH protocol is hierarchical and self-organizing cluster-based approach for monitoring applications based on Time Division Multiple Accesses (TDMA). New CHs are selected after specific time intervals. A node can be reselected only after all the remaining nodes have been elected. Lindsey and Raghavendra (2002) proposed PEGASIS is also an extension of LEACH. This routing protocol is having base station with short distance communication nodes from the geopolitical locations of nodes (Handy et al., 2002). Bandyopadhyay and Coyle (2003) described a multilevel hierarchical clustering algorithm to minimize the energy consumption and obtain using stochastic geometry. Zhu et al. (2009) proposed DEEC based on the ratio between residual energy of each node and the average energy of the network $\mathrm{CH}$ is selected. Selection of $\mathrm{CH}$ in a network is done based on the initial and residual energy of the nodes. The nodes having high initial and residual energy become a $\mathrm{CH}$. The new protocol that improves set-up phase of LEACH protocol is called LEACH-Centralized (LEACH-C). Central control algorithm is used to form the clusters, may produce better cluster design by assigning the $\mathrm{CH}$ nodes throughout the network. Hussain et al. (2007) introduced the Hierarchical Cluster based Routing (HCR) protocol (Zahmatkesh and Yaghmaee, 2012; Matin and Hussain, 2006). All the nodes are self-organized in to clusters and each cluster is managed by a set of associates called head-set and $\mathrm{CH}$ selected by Round Robin technique. Hussain et al. (2007) improved the HCR protocol by using a heuristic-based approach. It uses GA to determine the cluster, $\mathrm{CH}$ and $\mathrm{CH}$ members and the transmission schedule (Hussain et al., 2007). Maraiya et al. (2011) describe Energy Efficient Cluster Head Selection Scheme for Data Aggregation (ECHSSDA) protocol. It is having steady-state phase to improve the life time of the network (Maraiya et al., 2011). Jin and Sonenshein (1994) also used GA for energy optimization in WSN (Zahmatkesh and Yaghmaee, 2012). Formation of predefined independent clusters to reduce the total minimum communication distance. Ferentinos et al. (2005) extended the attempts proposed by Jin and Sonenshein (1994) by improving the GA fitness function (Matin and Hussain, 2006). The work done is based on the optimization properties of genetic algorithms. Cluster based routing protocol to minimize square sum of the distance between the node and the cluster head, or the centrality of the node can be recognized as an important factor to selecting cluster heads (Zhu et al., 2008; Ferentinos et al., 2005).

\section{PROPOSED SYSTEM}

The proposed mechanism based an adaptive clustering protocol to determine the optimal thresholding probability for cluster formation with GA in WSNs. 


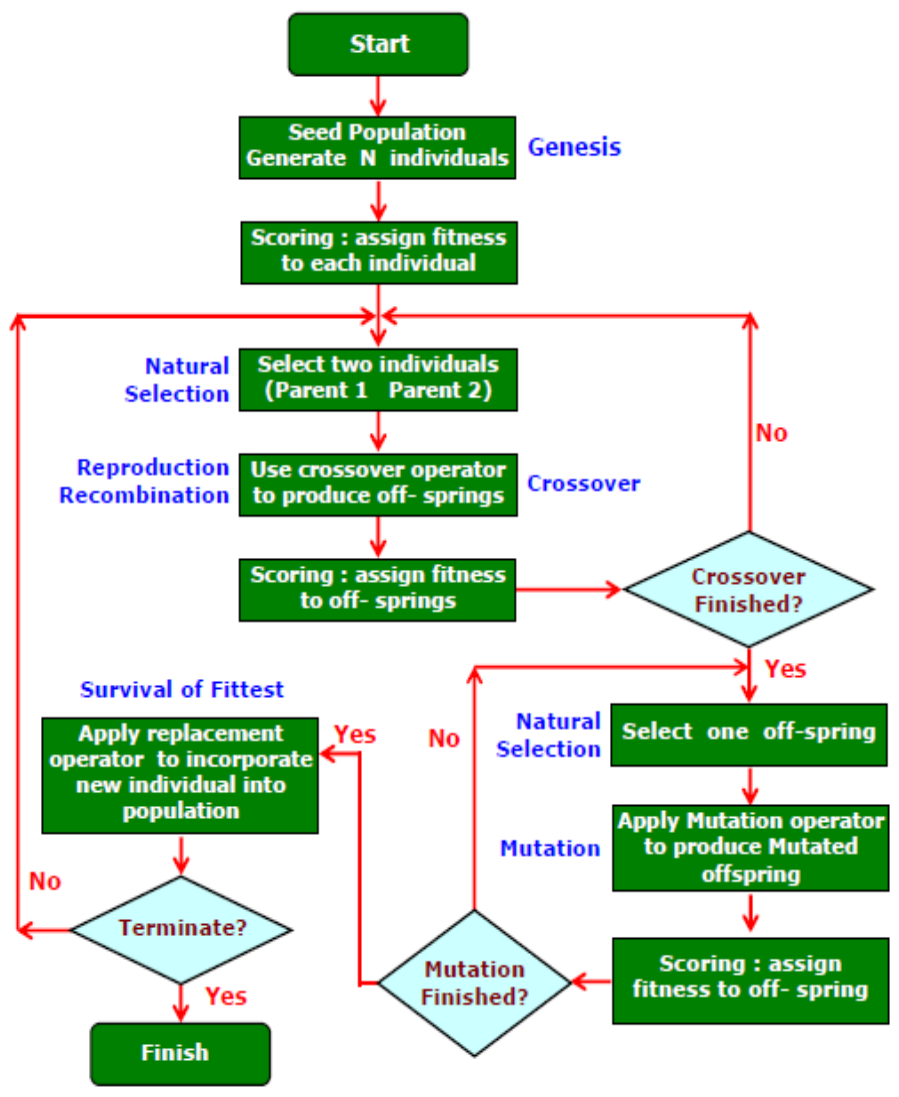

Fig. 2. shows the flow of proposed mechanism

This mechanism consists of set-up and steady-state phases for each round in the protocol and an additional preparation phase before the beginning of the first round. Preparation phase prior to the set-up phase of the first round to gather information about node status, IDs and location and sends it to the BS, which determines the optimal probability to use in the $\mathrm{CH}$ selection mechanism. Once the base station received the messages from all nodes, then it searches for an optimal probability of nodes being cluster heads via a genetic algorithm by minimizing the total energy consumption required for completing one round in the sensor field.

\subsection{Algorithm Steps for Genetic Leach}

Figure 2 shows the flow of proposed algorithm:

Step 1: Parameter values: Parameter values have been defined at first Table 1. The parameters used here are Sensing Area, Network size, Propagation model, Network interface, MAC interface, Seed, Interface queue type, Interface queue length, Antenna type, transport layer protocol, Application, Stop, MDC energy level, RX power, TX power, packet. These the parameters are defined at first in the program

Step 2: Global variables: Here values for each parameter is defined such as syntax, creation of topography for nodes and nodes position.

Step 3: Trace object for NS and NAM: Trace object is created here were named as trace and NAM trace. Normally this file act as a history for the running program and in trace definition we define topology for $\mathrm{X}$ and $\mathrm{Y}$ for area.

Step 4: Create GOD: It is called as General Operational Directory whose main function is to packet forwarding and receiving, en-queue and dequeues. Here it stores the information about all nodes so this makes routing between nodes easier. 
Step 5: Global node setting: Configuration of parameters is done in NAM window and bandwidth of MAC layer is also done and nodes are assigned to corresponding channels.

Step 6: Traffic model: For movement of nodes C.P and S.C file must be loaded. This file is already defined in TCL but for the movement of nodes at this stage it is loaded using puts command.

Step 7: Simulation ends: The end of stimulation time for nodes to stop communication is allocated here.

Step 8: Defining the graph values and execution of graph: Defining of graph is done here. $\mathrm{X}$ value and $\mathrm{Y}$ value for $(\mathrm{X}, \mathrm{Y})$ axis is defined according to movement of nodes. AWK format is used for generation of graph.

Step 9: Printing XY values: The values of trace fd, number of nodes, routing, $\mathrm{CP}$, seed, propagation and antenna is printed by using puts command then the nodes start the simulation and command is created for running the program.

\subsection{Parameter Table}

\subsection{NAM Window}

\subsubsection{Communication between the Clusters}

Figure 3 shows the observation from the above simulation that the ch4 senses the data and then it sends the sensed data to its neighboring cluster head ch3 and then the data is being sent to gateway through $\mathrm{CH} 3$

\subsubsection{Data Aggregation at the Base-Station}

Figure 4 shows all the data which are collected by the nodes are being delivered to the gateway and the gateway sends the aggregated data to the sink i.e., the base station in between this process the nodes are moved from one cluster to another cluster.

\subsection{Performance Evolution 4.4.1. Node mobility Vs Packet Drops}

Figure 5 shows the comparison graph between the earlier method and the proposed method is being done by comparing the packet drop during the transmission of data the proposed method performs better than the earlier method by reducing the packet loss in network.

\subsubsection{Node mobility Vs Packet Delivery Ratio}

Figure 6 shows the packet delivery ratio and the speed of the node movements were aggregated in the earlier method the speed and the packet delivery ratio decreases step by step and then it goes to minimum speed but in the proposed method there is slight variation compared to the earlier method and achieves a steady speed and constant packet delivery.

\subsubsection{Mobility Vs End to end Delay}

Figure 7 Shows the end to end delay in the earlier method is too high so the packet loss in the network will be high this situation is been handled carefully and the end to end delay is minimized in the proposed algorithm by reducing it. The energy of the nodes is maintained and the lifetime of the wireless sensor network is extended.

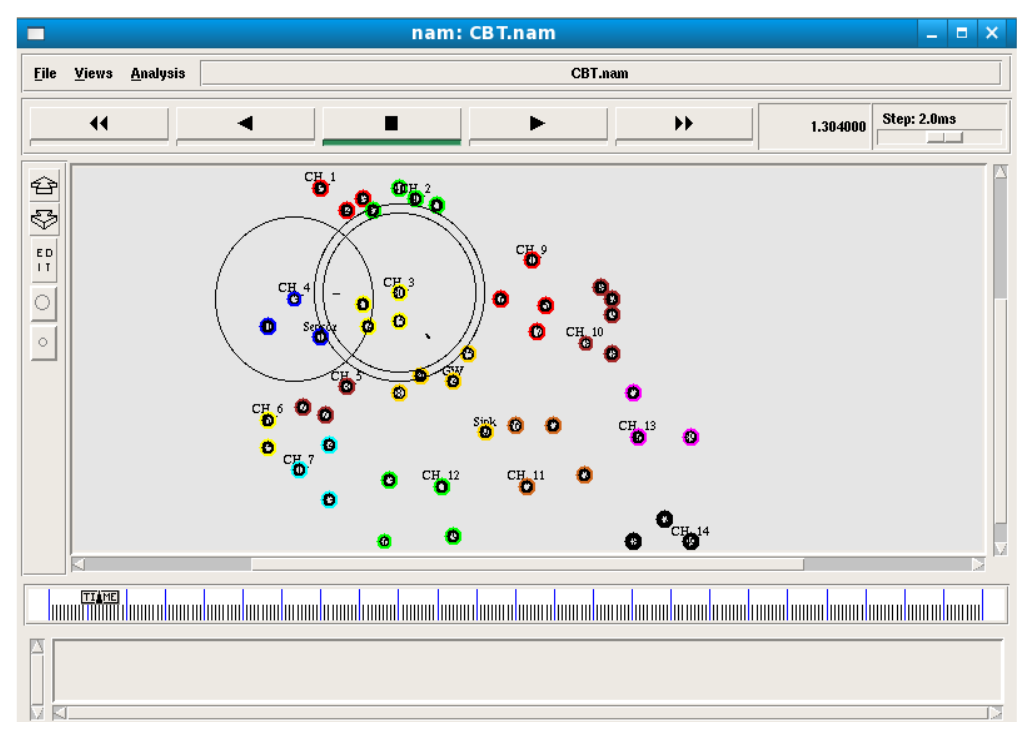

Fig. 3. Communication between the clusters 


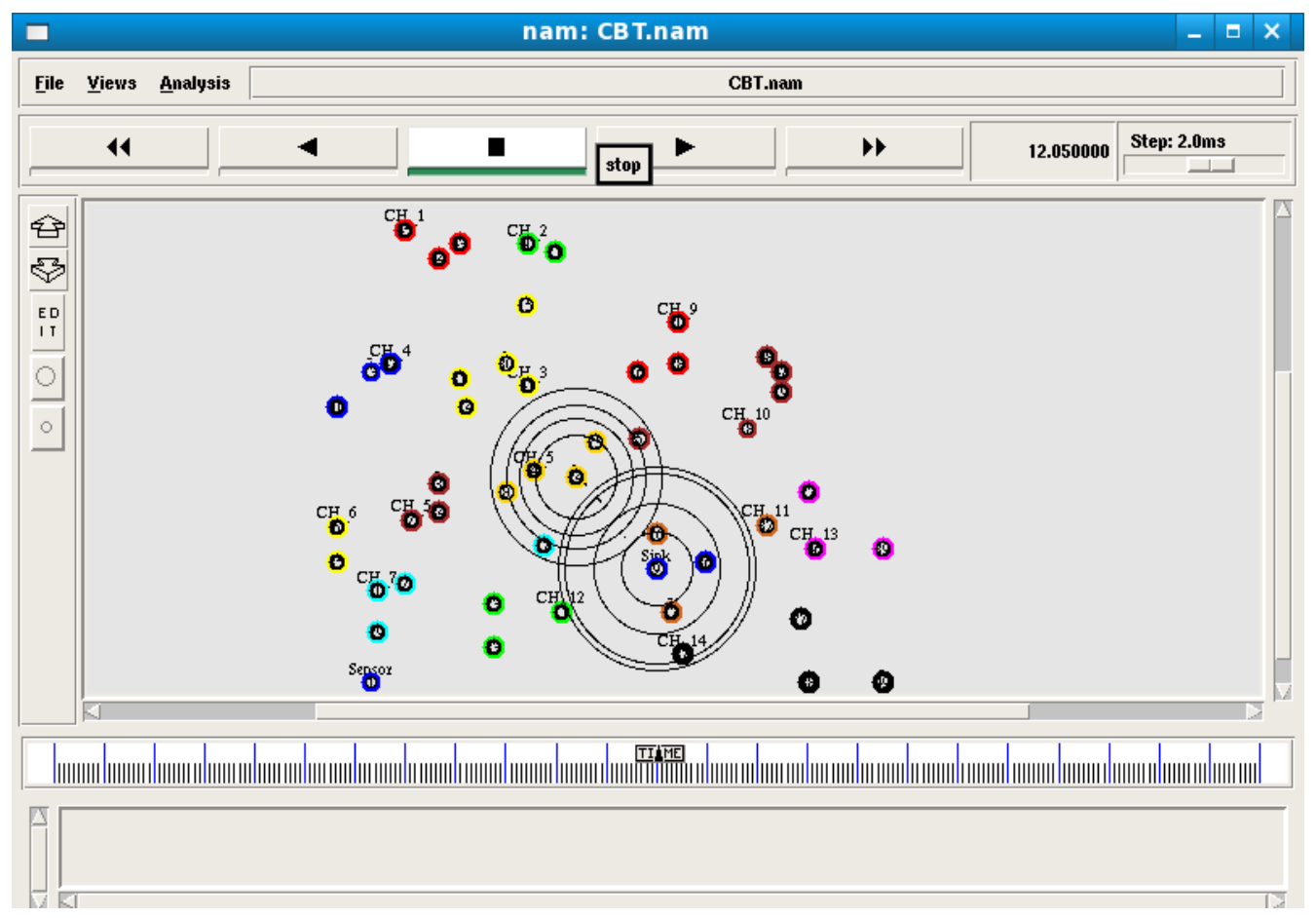

Fig. 4. Aggregation of data

\section{Q0Q xgraph}

Close Hdcpy About

Droppedpackets $\times 10^{3}$

NodeMobility_Vs packets_drops

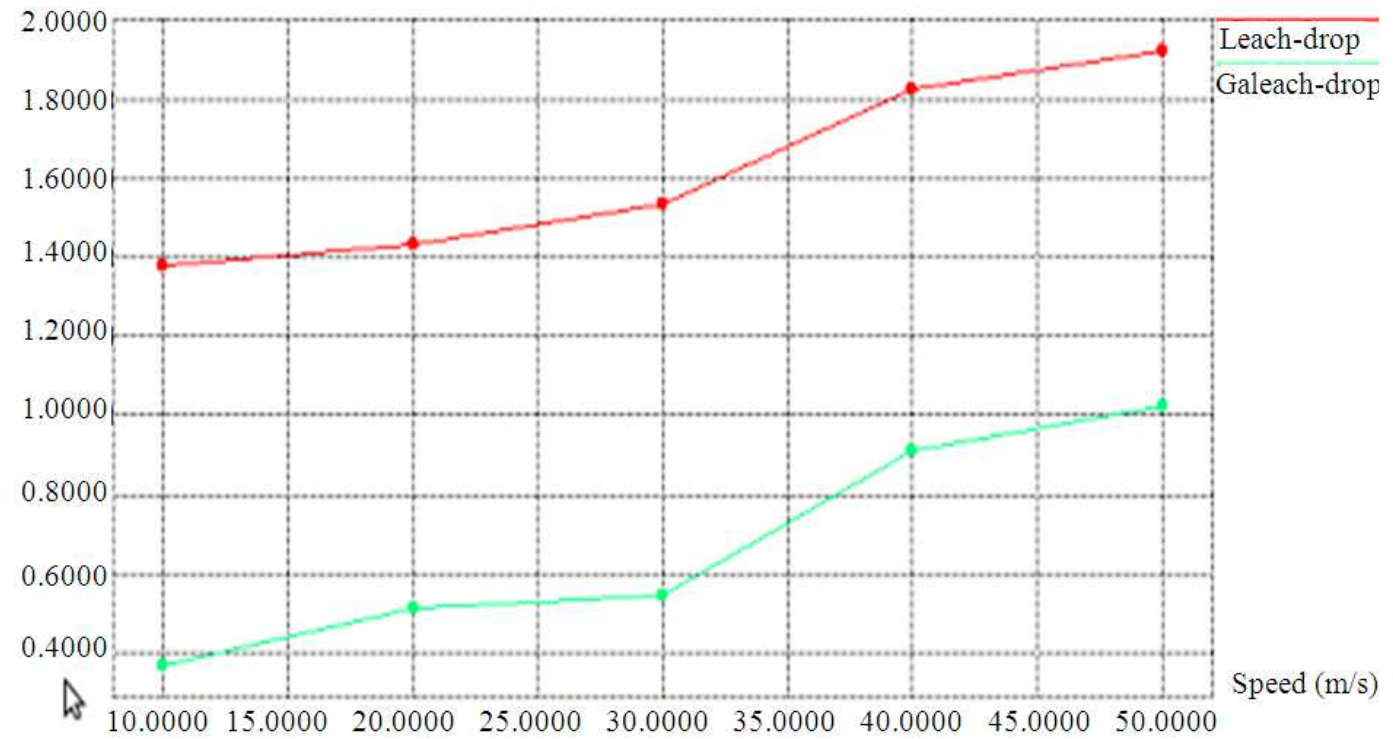

Fig. 5. Performance showing the packet loss during the node mobility 


\section{QQ $\mathrm{xgraph}$}

Close Hdopy About

Packet delivery ratio $\times 10^{-3}$

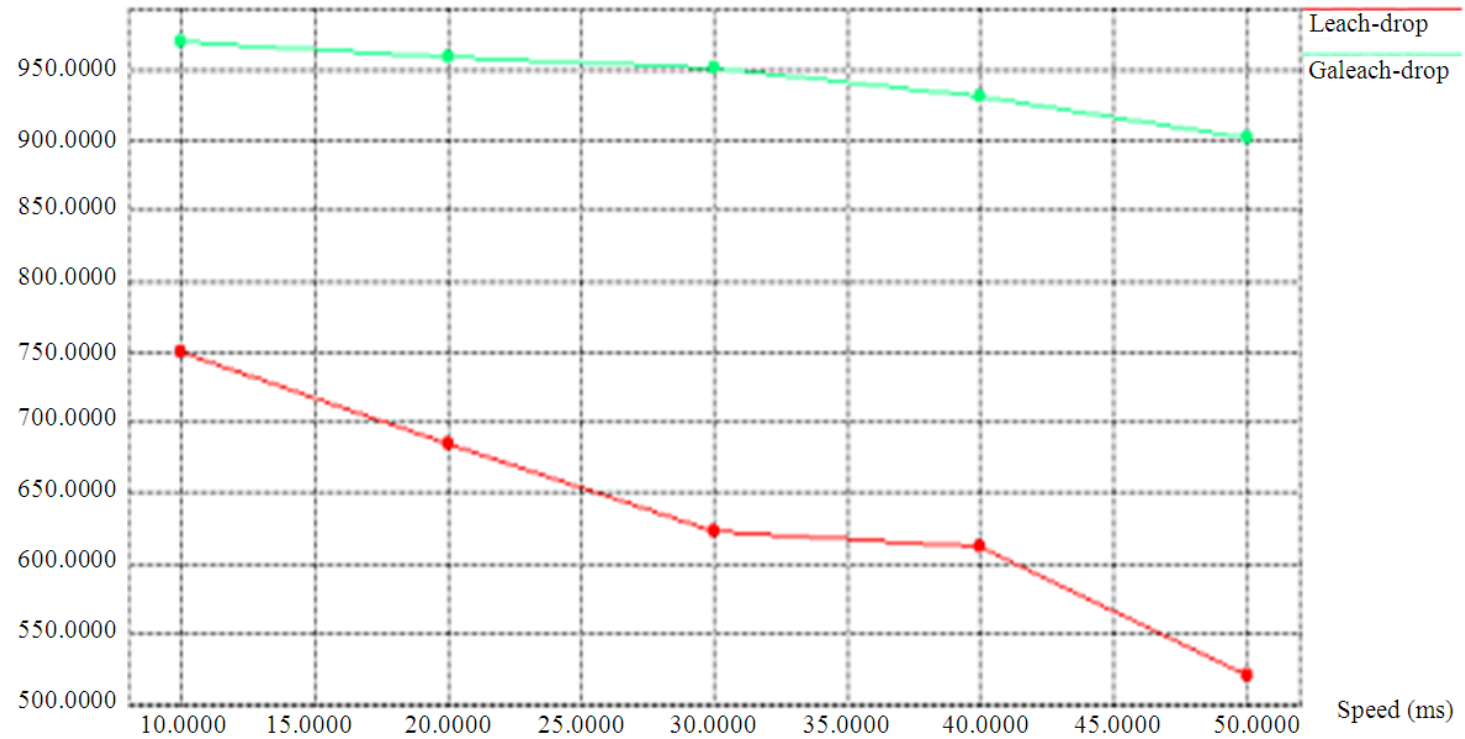

Fig. 6. Performance showing the packet delivery ratio during the node mobility

\section{QQ $\mathrm{Qgraph}$}

Close Hdcpy About

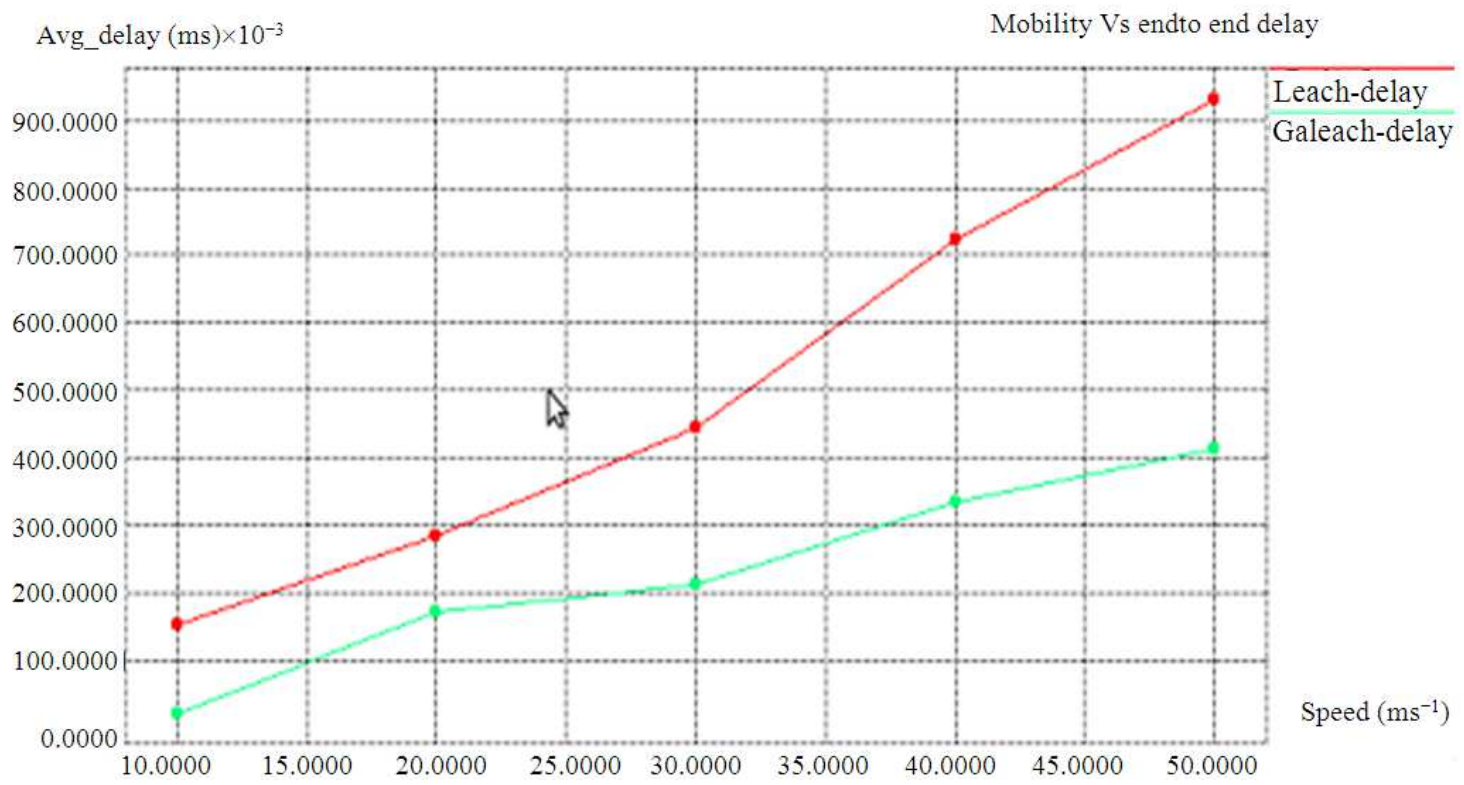

Fig. 7. Performance showing the end to end delays during the node mobility 


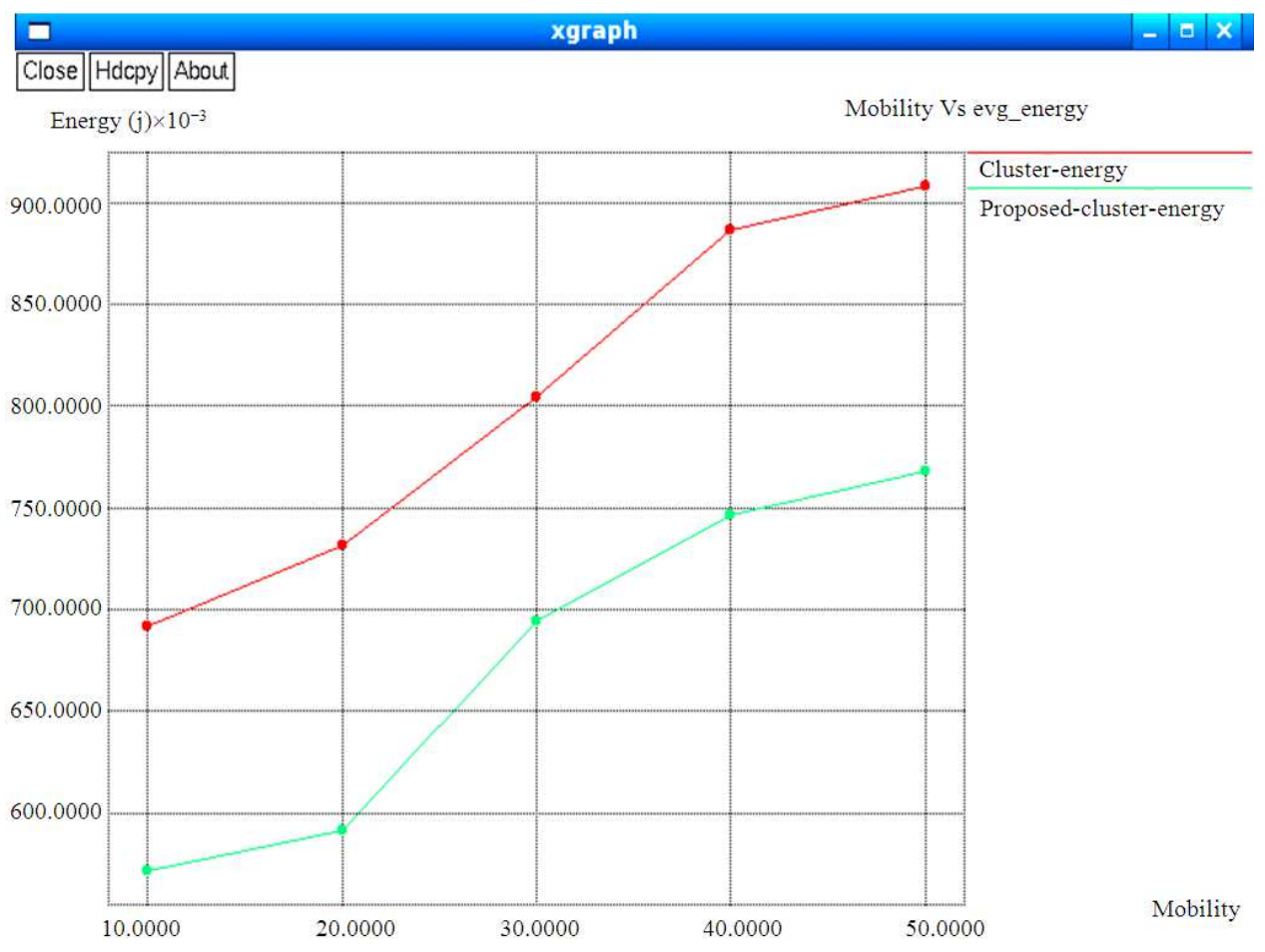

Fig. 8. Performance showing the Energy used during the node mobility

Table 1. Shows parameter values for proposed mechanism

\begin{tabular}{ll}
\hline Parameters & Value \\
\hline Area & $1000^{*} 1000$ \\
Channel & Channel/wireless channel \\
Propagation model & Propagation/Two ray ground \\
Network interface & Physical/wireless physical \\
MAC interface & Mac 802.11 ext \\
Seed & 0.0 \\
Interface queue type & Queue/Drop Tail/Pri Queue \\
Interface queue length & 50 \\
Antenna type & Antenna/Omni Antenna \\
Routing protocol & CBRP \\
transport layer protocol & TCP \\
Application & FTP \\
Stop & $50(\mathrm{~s})$ \\
\hline
\end{tabular}

\subsubsection{Mobility Vs Energy}

Figure 8 shows energy consumed by proposed mechanism is very less compared to the other protocol for $50 \mathrm{sec}$.

\section{CONCLUSION}

In proposed mechanism, to minimize the energy consumption of nodes introduced the genetic based energy efficient clustering algorithms. This Genetic algorithm is an adaptive method to search and optimize the energy consumption. The result shows Clustering is an effective way for reducing energy consumption of a sensor nodes as well as the cost of transmission. The proposed genetic algorithm based clustering protocol provide better efficiency in terms of delay, packet drop, packet delivery ratio.

\section{REFERENCES}

Akyildiz, I.F., W. Su, Y. Sankarasubramniam and E. Cayirei, 2002. A survey on sensor networks. IEEE Commun. Mag., 40: 102-114. DOI: 10.1109/MCOM.2002.1024422

Bandyopadhyay, S. and E.J. Coyle, 2003. An energy efficient hierarchical clustering algorithm for wireless sensor networks. Proceedings of the IEEE Conference on Computer Communications, Mar. 30Apr. 3, IEEE Xplore Press, pp: 1713-1723. DOI: 10.1109/INFCOM.2003.1209194

Ferentinos, K.P., T.A. Tsiligiridis and K.G. Arvanitis, 2005. Energy optimization of wireless sensor networks for environmental measurements. Proceedings of the International Conference on Computational Intelligence for Measurements Systems and Applicatons, Jul. 20-22, IEEE Xplore Press, pp: 250-255. DOI: 10.1109/CIMSA.2005.1522872 
Goldberg, D.E., 1989. Genetic Algorithms in Search, Optimization and Machine Learning, 1st Edn., Addison Wesley, Reading, MA., ISBN-10: 0201157675 , pp: 412.

Guo, L., Q. Li and F. Chen, 2011. A novel cluster-head selection algorithm based on hybrid genetic optimization for wireless sensor network. J. Network, 6: 815-822. DOI: 10.4304/jnw.6.5.815822

Handy, M.J., M. Haase and D. Timmermann, 2002. Low energy adaptive clustering hierarchy with deterministic cluster-head selection. Proceedings of the 4th IEEE Conference on Mobile and Wireless Communications Networks, IEEE Xplore Press, Stockholm, pp: 368-372. DOI: 10.1109/MWCN.2002.1045790

Heinzelman, W.R., A. Chandrakasan and H. Balakrishnan, 2000. Energy-efficient communication protocol for wireless microsensor networks. Proceedings of the 33rd Annual Hawaii International Conference on System Sciences, Jan. 4-7, IEEE Xplore Press, pp: 00-00. DOI: 10.1109/HICSS.2000.926982

Hussain, S., A.W. Matin and O. Islam, 2007. Genetic algorithm for energy efficient clusters in wireless sensor networks. Proceedings of the 4th International Conference on Information Technology, Apr. 2-4, IEEE Xplore Press, Las Vegas, NV., pp: 147-154. DOI: 10.1109/ITNG.2007.97

Jin, S. and A.L. Sonenshein, 1994. Transcriptional regulation of Bacillus subtilis citrate synthase genes. J. Bacteriol., 176: 4680-4690. PMID: 8045899
Lindsey, S. and C.S. Raghavendra, 2002. PEGASIS: Power-efficient gathering in sensor information systems. Proceedings of the IEEE Aerospace Conference, (AC' 02), IEEE Xplore Press, pp: 31125-3-1130. DOI: 10.1109/AERO.2002.1035242

Maraiya, K., K. Kant and N. Gupta, 2011. Efficient cluster head selection scheme for data aggregation in wireless sensor network. Int. J. Comput. Appli., 23: 10-18. DOI: $10.5120 / 2981-3980$

Matin, A.W. and S. Hussain, 2006. Intelligent hierarchical cluster-based routing. Proceedings of the International Workshop on Mobility and Scalability in Wireless Sensor Networks (MSWSN) in IEEE International Conference on Distributed Computing in Sensor Networks (DCOSS), pp: 165-172.

Younis, O. and S. Fahmy, 2004. HEED: A hybrid, energy-efficient, distributed clustering approach for ad hoc sensor networks. IEEE Trans. Mobile Comput., 3: 366-379. 10.1109/TMC.2004.41

Zahmatkesh, A. and M.H. Yaghmaee, 2012. A genetic algorithm-based approach for clustering of wireless sensor networks. Int. J. Inform. Electr. Eng., 2: .

Zhu, S., W. Wang and C.V. Ravishankar, 2008. PERT: A new power-efficient real-time packet delivery scheme for sensor networks. Int. J. Sensor Networks, 3: 237-251.

Zhu, X., L. Shen and T.S.P.Y. Hausdorff, 2009. Clustering and minimum energy routing for wireless sensor networks. IEEE Trans. Vehicular Technol., 58: 990-997. 